\title{
A NOVEL COARSE-MESH METHOD APPLIED TO NEUTRON SHIELDING PROBLEMS USING THE MULTIGROUP TRANSPORT THEORY IN DISCRETE ORDINATES FORMULATIONS
}

\author{
Libotte $^{1}$ R.B., Alves Filho ${ }^{1}$ H., Barros ${ }^{1}$ R.C. \\ ${ }^{1}$ Programa de Pós-graduação em Modelagem Computacional, 28625-570, Nova Friburgo, Rio de Janeiro, Brazil \\ rafaellibotte@hotmail.com \\ halves@iprj.uerj.br \\ rcbarros@pq.cnpq
}

\begin{abstract}
In this paper, we propose a new deterministic numerical methodology to solve the one-dimensional linearized Boltzmann equation applied to neutron shielding problems (fixed-source), using the transport equation in the discrete ordinates formulation $\left(S_{\mathrm{N}}\right)$ considering the multigroup theory. This is a hybrid methodology, entitled Modified Spectral Deterministic Method (SDM-M), that derives from the Spectral Deterministic Method (SDM) and Diamond Difference $(D D)$ methods. This modification in the $S D M$ method aims to calculate neutron scalar fluxes with lower computational cost. Two model-problems are solved using the $S D M-M$, and the results are compared to the coarse-mesh methods $S D M$, Spectral Green's Function $(S G F)$ and Response Matrix (RM), and the fine-mesh method $D D$. The numerical results were obtained in the programming language JAVA version 1.8.0_91.
\end{abstract}

Keywords: neutron transport theory, mathematical modelling, discrete ordinates, neutron shielding, fixedsource calculations, deterministic computational neutronic. 


\section{INTRODUCTION}

Neutron transport modelling have been a crescent research area, due to the many fields that need an accurate knowledge of the neutron flux behavior and it's interactions with the nuclei that constitutes the material zones, e.g., neutron shielding (fixed-source problems), radiology protection, nuclear medicine and oil prospecting. The calculation of the neutron angular flux inside a material zone can be computationally expensive when it comes to solve real world problems. Thus, the development of new methodologies to solve these problems accurately and in a faster way have been the main goal of our computational deterministic neutronic group.

A neutron moving inside a material zone can be described deterministically by the linearized Boltzmann equation, derived for the gas kinetics theory [1,2]. Which is a linear partial integrodifferential equation with 3 spatial variables, 2 angular variables, 1 time variable and 1 energetic variable. This equation represents a balance of production and loss of neutrons, assuming that the interaction of these particles with hosting media does not affect its structure, and there is no interaction between them. In this work, only stationary one-dimensional models are studied. The angular variable is treated according to the discrete ordinates formulation $\left(S_{N}\right)$, with multigroup method [1].

The spatial variable can be treated with coarse or fine-mesh methods. The solution of neutron transport problem using a fine-mesh approach, as for example Diamond Difference (DD) [1], can be mathematically simple, but it's usage can be very computationally expensive, once the mesh needs to be refined in order to achieve accurate results. On the other hand, coarse-mesh methods (nodal methods), e.g. Spectral Deterministic Method (SDM) [3,4], spectral Green's function (SGF) [5,6] and Response Matrix (RM) [7], may have a more complex implementation, but these methods are able to compute the angular fluxes inside large regions with good precision and without the need of any mesh refinement.

In this work, we propose a deterministic numeric method to solve the linearized Boltzmann equation applied to neutron shielding problems (fixed-source), denominated Modified Spectral Deterministic Method (SDM-M). This is a hybrid method that incorporates the spectral analysis used in the SDM to solve the neutron transport equation analytically in homogeneous regions 
(arbitrary nodes) with the iterative process found in the traditional method DD, known as Source Iteration (SI) [1], in order to solve the angular fluxes in node-edge. This modification in the iterative process is the main contribution of this work. Several tests are performed in order to find an iterative process with lower CPU time when executing model-problems.

Hereafter a brief summary of the content that compose this work is presented. In Section 2, the neutron transport equation mathematical modelling and its analytical solution are introduced. In Section 3, the Modified Spectral Deterministic Method's iterative process is proposed. In Section 4, the results of two model-problems using the SDM-M are analyzed and compared to the ones obtained with the SDM, SGF, RM, and DD. In Section 5, the results and comparisons presented in its previous section are discussed.

\section{MATHEMATICAL MODELLING OF NEUTRON TRANSPORT EQUATION}

The neutron transport equation in an one-dimensional domain $D$ with width $H$, stationary form, using the discrete ordinates formulations and $G$ groups of energy, has the form [1]

$$
\mu_{m} \frac{d}{d x} \psi_{m, g}(x)+\sigma_{T, g}(x) \psi_{m, g}(x)=\sum_{g^{\prime}=1}^{G} \frac{\sigma_{S 0}^{g^{\prime} \rightarrow g}(x)}{2} \sum_{n=1}^{N} \omega_{n} \psi_{n, g^{\prime}}(x)+Q_{g}(x), m=1: N, g=1: G
$$

where $\sigma_{T, g}(x)$ is the macroscopic total cross section in group g, the $\sigma_{S 0 j}^{g^{\prime} \rightarrow g}(x)$ is the zero-th order component of the macroscopic differential scattering cross section from group g' to group $g$ and $Q_{g}(x)$ is the isotropic fixed-source in group $g . \mu_{m}$ and $\omega_{m}$ are respectively the discrete directions and weights of the Gauss-Legendre quadrature of order N. The dependent variable $\psi_{m, g}(x)$ is the neutron angular flux of the $\mathrm{g}$ group. The Eq.(1) has prescribed boundary conditions given by 


$$
\psi_{m, g}(x)=\left\{\begin{array}{l}
b_{g}, \text { if } x=0 \text { and } \mu_{m}>0 \\
c_{g}, \text { if } x=H \text { and } \mu_{m}<0
\end{array}\right.
$$

This problem, represented in Eq. (1) may have a hard solution when considering $\sigma_{T, g}(x)$, $\sigma_{S 0}^{g^{\prime} \rightarrow g}(x)$ and $Q_{g}(x)$ as space-dependant variables. Thus, the spatial domain $D$ is divided into $J$ nodes $\left(\Gamma_{j}\right)$, as shown in Figure 1, with constant physical-material parameters

Figure 1: Discretized spatial domain.

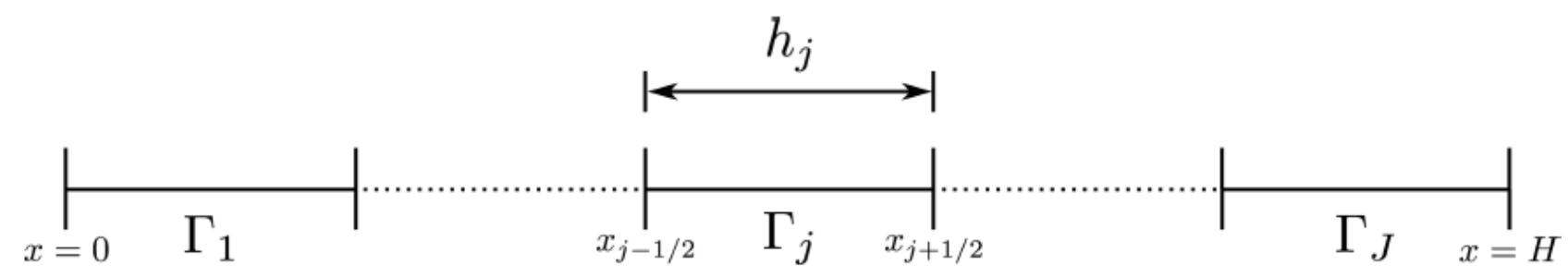

Source: Authors

Thus, Eq.(1) assumes the following form inside an arbitrary node $\Gamma_{j}$

$\mu_{m} \frac{d}{d x} \psi_{m, g}(x)+\sigma_{T, g, j} \psi_{m, g}(x)=\sum_{g^{\prime}=1}^{G} \frac{\sigma_{S 0, j}^{g^{\prime} \rightarrow g}}{2} \sum_{n=1}^{N} \omega_{n} \psi_{n, g^{\prime}}(x)+Q_{g, j}, m=1: N, g=1: G$

From Eq.(3), the analytical general solution can be determined in form [5,6]

$$
\psi_{m, g}(x)=\psi_{m, g}^{h}(x)+\psi_{m, g}^{p}, m=1: N, g=1: G
$$

The particular solution component $\left(\psi_{m, g}^{p}\right)$ is given by the system [3,4] 


$$
\sigma_{T, g, j} \psi_{m, g}^{p}-\sum_{g^{\prime}=1}^{G} \frac{\sigma_{S 0, j}^{g^{\prime} \rightarrow g}}{2} \sum_{n=1}^{N} \omega_{n} \psi_{n, g}^{p}=Q_{g, j}, m=1: N, g=1: G
$$

In order to obtain the homogeneous component, let us consider the expression [7]

$$
\psi_{m, g}^{h}(x)=a_{m, g}(\vartheta) e^{\frac{-(x-\eta)}{\vartheta}}, m=1: N, g=1: G
$$

where $\eta$ guarantees that the exponent will always assume negative values, avoiding numerical instability in model-problems. The parameter $\eta$ is defined as [7]

$$
\eta=\left\{\begin{array}{l}
x_{j-1 / 2}, \text { if } \vartheta>0 \\
x_{j+1 / 2}, \text { if } \vartheta<0
\end{array}\right.
$$

We emphasize that the displacement (espace shift) of the exponential argument derives from radiative transfer works and was adapted to the problems involving the neutron transport $[8,11]$.

Now, substituting Eq. (6) into the homogeneous part of Eq. (3) after some algebra, we obtain

$$
\frac{\delta_{m, n} \sigma_{T, g, j} a_{m, g}(\vartheta)}{\mu_{m}}-\sum_{g^{\prime}=1}^{G} \frac{\sigma_{S 0, j}^{g^{\prime} \rightarrow g}}{2 \mu_{m}} \sum_{n=1}^{N} \omega_{n} a_{n, g^{\prime}}(\vartheta)=\frac{1}{\vartheta} a_{m, g}(\vartheta), m=1: N, g=1: G,
$$

where $\delta_{m n}$ represents Kronecker's delta. Equation (8) can be written in a compact form

$$
\boldsymbol{A a}(\vartheta)=\frac{1}{\vartheta} \boldsymbol{a}(\vartheta)
$$

Solving the system presented in the Eq.(9), $\mathrm{N}$ real symmetric eigenvalues $( \pm \vartheta)$ are determined. Thus, for each arbitrary node $\Gamma_{j}$ a set of $\mathrm{N}$ linearly independent eigenfunctions is obtained. The intra-nodal solution given by Eq.(3) takes the form 


$$
\psi_{m, g}(x)=\sum_{l=1}^{N G} \alpha_{l} a_{m, g}\left(\vartheta_{l}\right) e^{\frac{-(x-\eta)}{\vartheta_{l}}}+\psi_{m, g}^{p}, m=1: N, g=1: G
$$

where $\boldsymbol{\alpha}$ is a set of arbitrary parameters to be defined in each node.

\section{MODIFIED SPECTRAL DETERMINISTIC METHOD}

In this section, we present the method Spectral Deterministic Method (SDM-M), which is the main contribution of this work. The solution of the neutron transport equation using this method is based on two main points: the $\alpha$ parameters as shown in Eq. (10) $[3,4]$ and an iterative process to calculate the angular fluxes, based on a modification of the Source Iteration method (SI) [1]. In order to illustrate the iterative process that calculates the neutron angular fluxes in each node, an example with 3 spatial nodes, based on the spatial grid in shown in Figure 2. The black solid arrows represent the boundary conditions and the gray dotted lines represents the initial estimates.

Figure 2: Iterative process - initial condition.

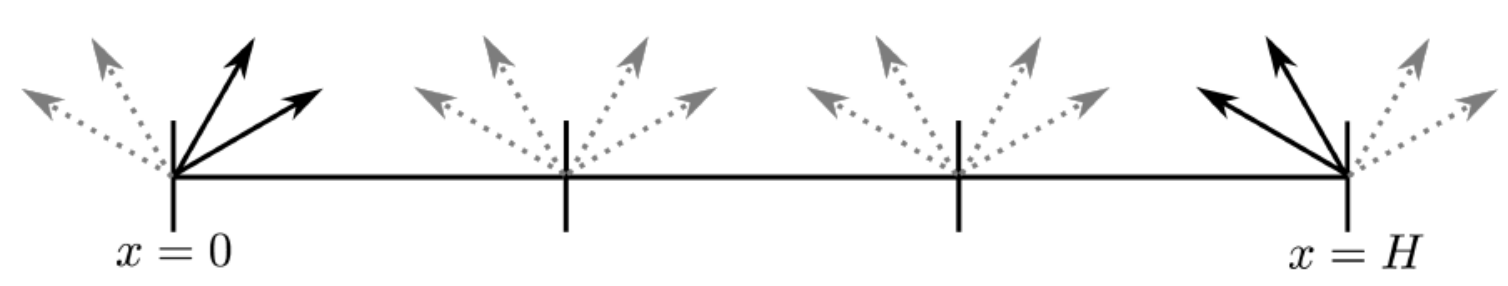

Source: Authors

Once the initial estimates and the boundary conditions are defined, the first step to start the iterative process is to calculate $\alpha_{l}$, Eq. (10), these parameters can be determined by using the incoming neutron angular fluxes in the first node [3,4]. With these values, the next step is to calculate the average neutron angular flux with 


$$
\overline{\psi_{j}}=\frac{1}{h_{j}} \int_{x_{j-1 / 2}}^{x_{j+1 / 2}} \psi_{m, g}(x) \mathrm{d} x, m=1: N, g=1: G .
$$

Now, subistitutuing $\psi_{m, g}(x)$ from Eq. (10) into Eq. (11), we obtain the homogeneous components

$$
\begin{aligned}
& \frac{-1}{h_{j}} \alpha \vartheta a_{m, g}(\vartheta)\left(e^{-h_{j} / \vartheta}-1\right) \text {, if } \vartheta>0, m=1: N, g=1: G, \\
& \frac{-1}{h_{j}} \alpha \vartheta a_{m, g}(\vartheta)\left(1-e^{h_{j} / \vartheta}\right) \text {, if } \vartheta<0, m=1: N, g=1: G .
\end{aligned}
$$

Now, we set up a computational algorithm to obtain the outgoing node-edge angular fluxes $\left(\psi_{m, g, j \pm 1 / 2}^{\text {out }}\right)$ of a $\Gamma_{j}$ node, knowing the incoming node-edge angular fluxes $\left(\psi_{m, g, j \pm 1 / 2}^{\text {in }}\right)$ and the fixed-source of neutrons $\left(Q_{g, j}\right)$ inside this node, An illustrative scheme is presented in Figure 3.

Figure 3: Representation of the incoming and outgoing node-edge neutron angular fluxes.

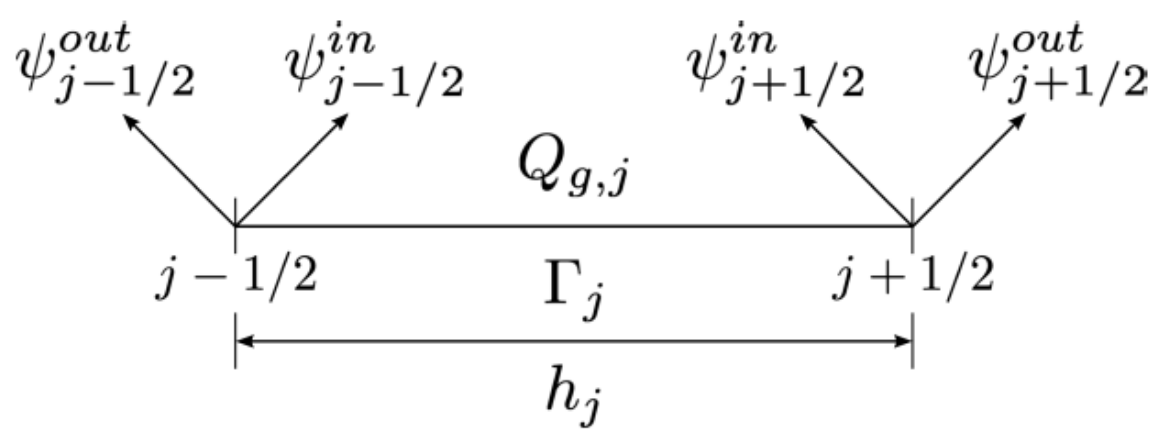

Source: Authors

The iterative process is similar to the one used in SDM $[3,4]$. We define as an iteration the course in the spatial domain seen in Figure 2, moving over all the nodes. Starting in $\mathrm{x}=0$ until , $\mathrm{x}=\mathrm{H}$ calculating all the outgoing node-edge angular fluxes $\psi_{m, g, j \pm 1 / 2}^{\text {out }}$. The mathematical structure of these calculations, starts by applying the operator 


$$
\frac{1}{h j} \int_{x_{j-1 / 2}}^{x_{j+1 / 2}}(.) \mathrm{d} x
$$

in Eq. (3), considering the arbitrary node $\Gamma_{j}$ (Figure 3), we obtain the expression

$$
\begin{aligned}
& \frac{\mu_{m}}{h_{j}}\left(\psi_{m, g, j+1 / 2}-\psi_{m, g, j-1 / 2}\right)+\sigma_{T, g, j} \bar{\psi}_{j}= \\
& \sum_{g^{\prime}=1}^{G} \frac{\sigma_{S 0, j}^{g^{\prime} \rightarrow g}}{2} \sum_{n=1}^{N} \omega_{n} \bar{\psi}_{j}+Q_{g, j}, m=1: N, g=1: G .
\end{aligned}
$$

After a little algebra, we obtain

$$
\begin{array}{r}
\psi_{m, g, j+1 / 2}=\frac{h j}{\mu_{m}}\left(-\sigma_{T, g, j} \bar{\psi}_{g, j}+\sum_{g^{\prime}=1}^{G} \frac{\sigma_{S 0, j}^{g^{\prime} \rightarrow g}}{2} \sum_{n=1}^{N} \omega_{n} \bar{\psi}_{g, j}+Q_{g, j}\right)+\psi_{m, g, j-1 / 2}, \\
m=1: N / 2, g=1: G,
\end{array}
$$

for $\mu_{m}>0$, and

$$
\begin{array}{r}
\psi_{m, g, j-1 / 2}=\frac{h j}{\left|\mu_{m}\right|}\left(-\sigma_{T, g, j} \bar{\psi}_{g, j}+\sum_{g^{\prime}=1}^{G} \frac{\sigma_{S 0, j}^{g^{\prime} \rightarrow g}}{2} \sum_{n=1}^{N} \omega_{n} \bar{\psi}_{g, j}+Q_{g, j}\right)+\psi_{m, g, j+1 / 2}, \\
m=N / 2+1: N, g=1: G .
\end{array}
$$

to $\mu_{m}<0$.

When the outgoing node-edge neutron angular fluxes of the last node is computed, we perform the calculation of the neutron scalar flux $\left(\phi_{g}(x)\right)$ in every node interface (one interation) using the expression

$$
\phi_{\boldsymbol{g}}(x)=\frac{1}{2} \sum_{n=1}^{N} \psi_{n, g}(x) \omega_{n}
$$


This iterative process is performed until the maximum value of the norm for the relative deviation between two consecutives estimates for the scalar flux energy group $\varphi_{g}(x)$ does not exceed a pre-estabilished value. The relative deviation is calculated using the expression

$$
\max _{j=1: J+1}\left|\frac{\phi_{g, j-1 / 2}^{i}-\phi_{g, j-1 / 2}^{i-1}}{\phi_{g, j-1 / 2}^{i-1}}\right| \times 100 \%<\xi
$$

the variable $\xi$ is a pre-established value.

\section{NUMERICAL RESULTS}

In this section, we present the results of two model-problems to validate the SDM-M method. In both cases, the numerical results of SDM-M are compared to the traditional fine-mesh DD and the coarse-mesh methods SDM, SGF and RM. In the first model-problem, let us consider an homogeneous domain with 2 groups of energy and $100 \mathrm{~cm}$ of width. The physical-material parameters and the boundary conditions are displayed in Figure 4.

\section{Figure 4: Model-problem 1}

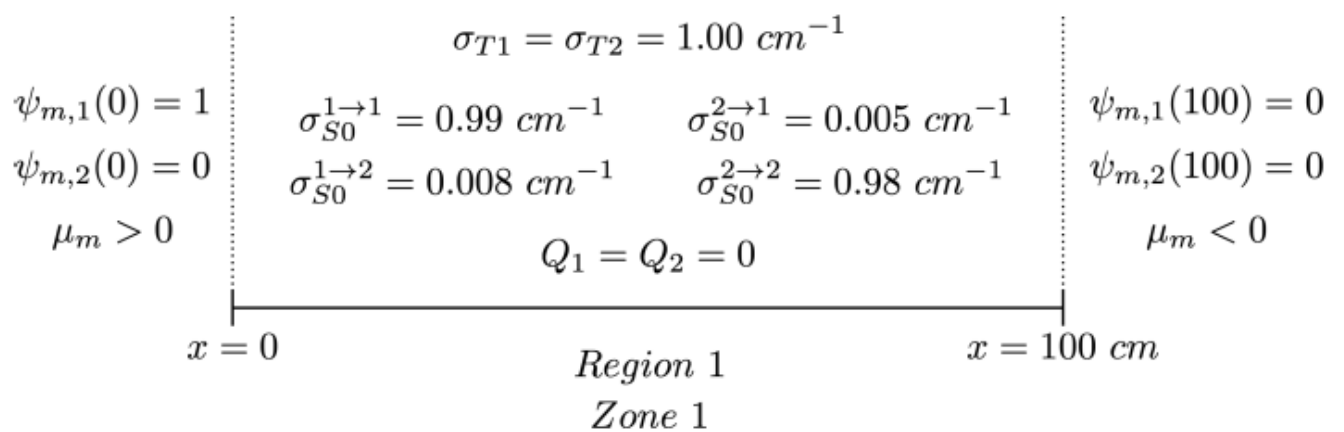

Source: Author

In model-problem 1 we use the Gauss-Legendre [1] quadrature of orders $\mathrm{N}=4$ and $\mathrm{N}=16$ and set $\xi=10^{-6}$. The $D D$ method uses a mesh with 500 nodes. The SDM-M and SDM methods 
were executed with 1 node. The results of the $S G F$ and $R M$ methods were taken from Ref. [3], which also simulates the same problem with 1 node per region. The scalar fluxes in the region interfaces for both groups are shown in Table 1.

Table 1: Scalar Fluxes $\left(\mathrm{cm}^{-2} s^{-1}\right)$

\begin{tabular}{|c|c|c|c|c|c|}
\hline \multirow[b]{2}{*}{ Method } & \multirow[b]{2}{*}{ Group } & \multicolumn{2}{|c|}{$N=4$} & \multicolumn{2}{|c|}{$N=16$} \\
\hline & & $\mathbf{x}=0 \mathrm{~cm}$ & $x=100 \mathrm{~cm}$ & $x=0 \mathrm{~cm}$ & $x=100 \mathrm{~cm}$ \\
\hline \multirow{2}{*}{$\mathrm{DD}^{\mathrm{a}}$} & 1 & $9.12675 \mathrm{E}-1^{\mathrm{c}}$ & $6.27693 \mathrm{E}-8$ & $9.12674 \mathrm{E}-1$ & $6.23009 \mathrm{E}-8$ \\
\hline & 2 & $2.72638 \mathrm{E}-2$ & $3.83316 \mathrm{E}-8$ & $2.72636 \mathrm{E}-2$ & $3.81557 \mathrm{E}-8$ \\
\hline \multirow{2}{*}{ SDM-M ${ }^{b}$} & 1 & $9.12675 \mathrm{E}-1$ & $6.27693 \mathrm{E}-8$ & $9.12675 \mathrm{E}-1$ & $6.24932 \mathrm{E}-8$ \\
\hline & 2 & $2.72639 \mathrm{E}-2$ & $3.84425 \mathrm{E}-8$ & $2.72639 \mathrm{E}-2$ & $3.82734 \mathrm{E}-8$ \\
\hline \multirow{2}{*}{$\mathrm{SDM}^{\mathrm{b}}$} & 1 & $9.12675 \mathrm{E}-1$ & $6.27693 \mathrm{E}-8$ & $9.12675 \mathrm{E}-1$ & $6.24932 \mathrm{E}-8$ \\
\hline & 2 & 2.72639E-2 & $3.84425 \mathrm{E}-8$ & $2.72639 \mathrm{E}-2$ & 3.82734E-8 \\
\hline \multirow{2}{*}{$\mathrm{SGF}^{\mathrm{b}}$} & 1 & $9.12675 \mathrm{E}-1$ & $6.27702 \mathrm{E}-8$ & $9.12675 \mathrm{E}-1$ & $6.24922 \mathrm{E}-8$ \\
\hline & 2 & 2.72639E-2 & $3.84431 \mathrm{E}-8$ & $2.72636 \mathrm{E}-2$ & 3.82667E-8 \\
\hline \multirow{2}{*}{$\mathrm{RM}^{\mathrm{b}}$} & 1 & $9.12675 \mathrm{E}-1$ & $6.27692 \mathrm{E}-8$ & $9.12675 \mathrm{E}-1$ & $6.24932 \mathrm{E}-8$ \\
\hline & 2 & $2.72639 \mathrm{E}-2$ & $3.84425 \mathrm{E}-8$ & $2.72639 \mathrm{E}-2$ & $3.82734 \mathrm{E}-8$ \\
\hline
\end{tabular}

${ }^{\mathrm{a}} 500$ nodes in region 1 .

${ }^{\mathrm{b}} 1$ node in region 1 .

${ }^{\mathrm{c}} \operatorname{Read} 9.12675 \times 10^{-1}$

The second test measures the average execution time of the SDM, SDM-M and DD methods in the solution of the model-problem 1, with quadrature order $\mathrm{N}=64$ and $\mathrm{N}=256$. Here, it should be noted that the scalar fluxes generated by these set order quadrature are approximately the same as shown in Table 1. This test consists in the execution of each method solving this model-problem 1000 times, storing each execution time and calculating its average. The results regarding the average execution time and iterations number, considering each method, is shown in Tables 2 and 3.

Table 2: Execution average time performance $(\mathrm{N}=64)$

\begin{tabular}{lll}
\hline Method & Average Time \pm Standard Deviation & Total \\
\hline
\end{tabular}




\begin{tabular}{ccc}
\hline & & Iteration \\
\hline DD & $3.462484 \pm 0.214295$ & 1225 \\
\hline SDM & $0.034948 \pm 0.028142$ & 2 \\
\hline SDM-M & $0.033017 \pm 0.024087$ & 2 \\
\hline
\end{tabular}

Table 3: Execution average time performance $(\mathrm{N}=256)$

\begin{tabular}{ccc}
\hline Method & Average Time \pm Standard Deviation & $\begin{array}{c}\text { Total } \\
\text { Iteration }\end{array}$ \\
\hline DD & $17.455859 \pm 1.212007$ & 1225 \\
\hline SDM & $2.206409 \pm 0.221716$ & 2 \\
\hline SDM-M & $2.085199 \pm 0.300421$ & 2 \\
\hline
\end{tabular}

The results obtained in the performance test for both Gauss-Legendre quadrature orders in the first model-problem, shows that the modification in the iterative process of the $S D M-M$ method provided a lower CPU time when compared to the $S D M$ and $D D$.

In second model-problem, let us consider a domain with 4 regions and 3 distinct material zones. Figure 5 shows the physical-material parameters and the boundary conditions.

Figure 5: Model-problem 2.

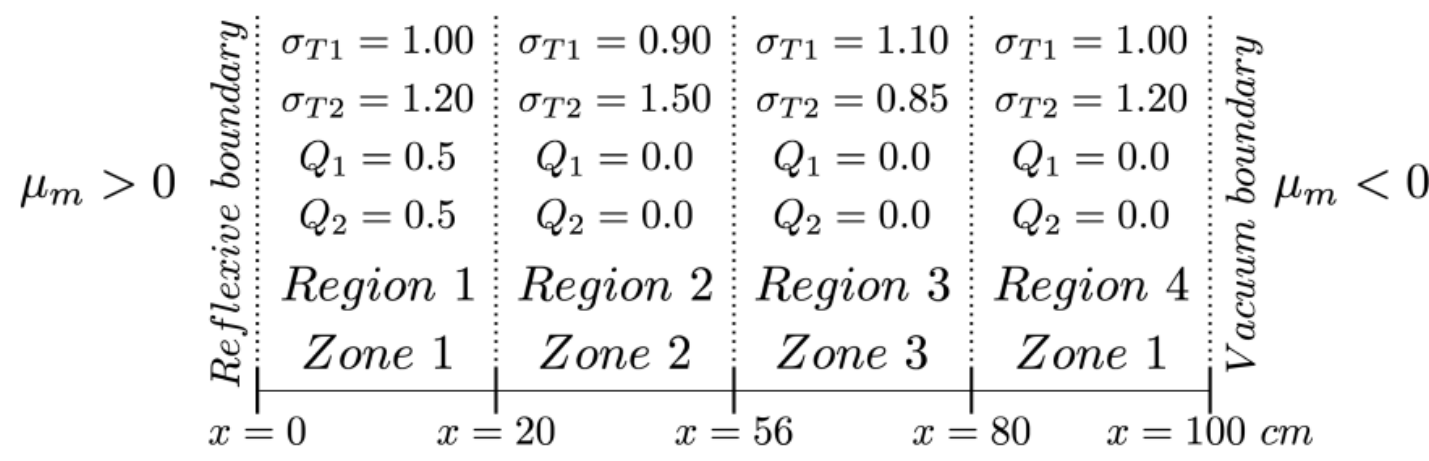

Source: Author

The isotropic scattering cross sections $\left(\sigma_{S 0}^{g \rightarrow g}\right)$ that compose the material zones in this problem are shown in Table 4. 
Table 4: Isotropic scattering macroscopic cross sections $\left(\sigma_{S 0}^{g^{\prime} \rightarrow g}\right)$.

\begin{tabular}{lcllclc}
\hline \multirow{2}{*}{ Method } & \multicolumn{2}{c}{ Zone 1 } & \multicolumn{2}{c}{ Zone 2 } & \multicolumn{2}{c}{ Zone 3 } \\
& $\mathrm{g}=1$ & $\mathrm{~g}=2$ & $\mathrm{~g}=1$ & $\mathrm{~g}=2$ & $\mathrm{~g}=1$ & $\mathrm{~g}=2$ \\
\hline$\sigma_{S 0}^{1 \rightarrow g}$ & 0.90 & 0.20 & 0.75 & 0.30 & 0.95 & 0.60 \\
\hline$\sigma_{S 0}^{2 \rightarrow g}$ & 0.05 & 0.80 & 0.10 & 0.99 & 0.00 & 0.20 \\
\hline
\end{tabular}

This problem was solved using the Gauss-Legendre quadrature of order $\mathrm{N}=32$, and $\xi=10^{-6^{-}}$.

To execute the $D D$ method, we used a mesh with 200 nodes in the first region, 360 in the second, 240 in the third and 200 on the fourth one. All the coarse-mesh methods used 1 node per region. Similarly as model-problem 1, the results for the $S G F$ and $R M$ methods were taken from Ref. [3]. The scalar fluxes for this problem are shown in Table 5.

Table 5: Scalar Fluxes $\left(\mathrm{cm}^{-2} \mathrm{~s}^{-1}\right)$

\begin{tabular}{|c|c|c|c|c|c|c|}
\hline \multirow{2}{*}{ Method } & \multirow{2}{*}{ Group } & \multicolumn{5}{|c|}{$\mathbf{N}=32$} \\
\hline & & $x=0 \mathrm{~cm}$ & $x=20 \mathrm{~cm}$ & $x=56 \mathrm{~cm}$ & $x=80 \mathrm{~cm}$ & $x=100 \mathrm{~cm}$ \\
\hline \multirow{2}{*}{$\mathrm{DD}^{\mathrm{a}}$} & 1 & $7.49899 \mathrm{E}+0^{\mathrm{c}}$ & $3.51557 \mathrm{E}+0$ & $1.86740 \mathrm{E}-7$ & $2.68910 \mathrm{E}-14$ & $1.50010 \mathrm{E}-18$ \\
\hline & 2 & $4.99941 \mathrm{E}+0$ & $2.55794 \mathrm{E}+0$ & $1.36574 \mathrm{E}-7$ & $3.41163 \mathrm{E}-14$ & 7.85261E-19 \\
\hline \multirow{2}{*}{ SDM-M ${ }^{\mathrm{b}}$} & 1 & $7.49903 \mathrm{E}+0$ & $3.51557 \mathrm{E}+0$ & $1.86413 \mathrm{E}-7$ & $2.72811 \mathrm{E}-14$ & $1.60046 \mathrm{E}-18$ \\
\hline & 2 & $4.99943 \mathrm{E}+0$ & $2.55794 \mathrm{E}+0$ & $1.37074 \mathrm{E}-7$ & $3.45835 \mathrm{E}-14$ & 8.38952E-19 \\
\hline \multirow{2}{*}{$\mathrm{SDM}^{\mathrm{b}}$} & 1 & $7.49902 \mathrm{E}+0$ & $3.51557 \mathrm{E}+0$ & $1.86413 \mathrm{E}-7$ & $2.72811 \mathrm{E}-14$ & $1.60046 \mathrm{E}-18$ \\
\hline & 2 & 4.99943E+0 & $2.55794 \mathrm{E}+0$ & $1.37074 \mathrm{E}-7$ & 3.45835 E-14 & 8.38952E-19 \\
\hline \multirow{2}{*}{$\mathrm{SGF}^{\mathrm{b}}$} & 1 & $7.49898 \mathrm{E}+0$ & $3.51553 \mathrm{E}+0$ & $1.86408 \mathrm{E}-7$ & $2.72790 \mathrm{E}-14$ & $1.60056 \mathrm{E}-18$ \\
\hline & 2 & $4.99940 \mathrm{E}+0$ & $2.55798 \mathrm{E}+0$ & $1.37052 \mathrm{E}-7$ & $3.45813 \mathrm{E}-14$ & 8.38935E-19 \\
\hline \multirow{2}{*}{$\mathrm{RM}^{\mathrm{b}}$} & 1 & 7.49898E+0 & $3.51554 \mathrm{E}+0$ & $1.86416 \mathrm{E}-7$ & $2.72807 \mathrm{E}-14$ & $1.60069 \mathrm{E}-18$ \\
\hline & 2 & $4.99941 \mathrm{E}+0$ & $2.55800 \mathrm{E}+0$ & $1.37057 \mathrm{E}-7$ & $3.45833 \mathrm{E}-14$ & 8.39003E-19 \\
\hline
\end{tabular}

${ }^{\mathrm{a}} 200$ nodes in region 1, 360 in region 2, 240 in region 3 and 200 in region 4.

$\mathrm{b} 1$ node per region.

${ }^{\mathrm{c}}$ Read as $7.49899 \times 10^{0}$

Figure 6 presents the numerical results in Table5 in graphic format to scalar fluxes based in model-problem 2. 
Figure 6: Scalar fluxes.

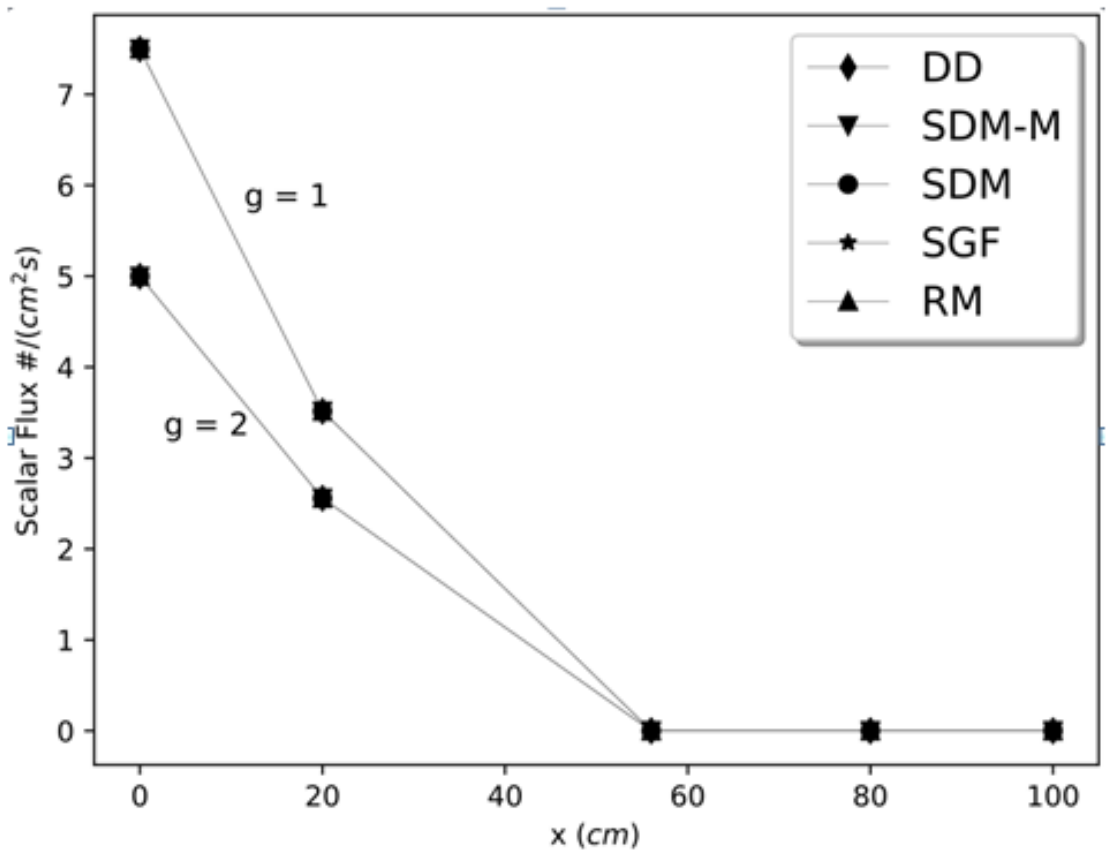

Source: Author

In this model-problem, the algorithm was executed 1000 times for each method, with quadrature order $N=128$. The results of the average execution time and the number of iterations are shown in Table 6. Again, the scalar fluxes generated by these set order quadrature are approximately the same as shown in Table 5, with $\mathrm{N}=32$.

Table 6: Execution average time performance $(\mathrm{N}=128)$

\begin{tabular}{ccc}
\hline Method & Average Time \pm Standard Deviation & Total Iteration \\
\hline DD & $14.142665 \pm 0.296941$ & 549 \\
\hline
\end{tabular}




\begin{tabular}{ccc} 
SDM & $3.267299 \pm 0.165041$ & 2 \\
\hline SDM-M & $3.027089 \pm 0.150199$ & 2 \\
\hline
\end{tabular}

As in the previous model-problem, SDM-M presented lower CPU time compared to the others methods. The numerical results presented in the problem-models 1 and 2, shows that SDM-M can archieve the same accuracy as the fine-mesh methods DD and the coarse-mesh method SDM, SGF and RM.

\section{CONCLUSIONS}

In this work, a new methodology to calculate neutron angular fluxes in shielding problems was proposed, called Modified Spectral Deterministic Method, using the linearized Boltzmann equation in the discrete ordinates formulation, considering multigroup, one-dimensional and stationary form. The results were compared to the coarse-mesh methods $S D M, R M$ and $S G F$, and the fine-mesh method $D D$.

In the first model-problem, $S D M-M$ obtained relatively accurate results for both Gaussianquadrature orders used in the solution, within the predefined threshold adopted for the iterative process. As in model-problem 1, the $S D M-M$ obtained accurate results for the neutron scalar fluxes, when compared to the other methods.

In this paper, we are concerned with investigating the accuracy and computational cost of the analyzed algorithm, which provides an alternative form of solution of the neutron transport equation in the discrete ordinates formulation, in comparison with the coarse-mesh method SDM. Based on the presented numerical results, it is concluded that: $S D M-M$ presents almost the same precision of the numerical results obtained by SDM. Second, the CPU time required to compute the results using $S D M-M$ is slightly lower, in comparison with $S D M$. Thus, the differences between the two methods, regarding the performance, will need further analysis.

For future works, we intend to implement an arbitrary degree of scattering cross section in the method, and study the absortion rate and the neutron leakage.

\section{ACKNOWLEDGMENT}


This study was financed in part by the Coordenação de Aperfeiçoamento de Pessoal de Nível Superior - Brasil (CAPES) - Finance Code 001. The group would like to thank the Laboratório de Modelagem Multiescala e Transporte de Partículas (LabTran).

\section{REFERENCES}

1. LEWIS, E. E.; MILLER, W. F. Computational methods of neutron transport. Wiley, New York \& United States of America, 1993.

2. DUDESTADT, J. J.; HAMILTON, L. J. Nuclear reactor analysis. Wiley, New York \& United States of America, 1976.

3. OLIVA, A. M. Método Espectral Determinístico para a solução de problemas de transporte de nêutrons usando a formulação das ordenadas discretas, Tese de Doutorado, Universidade do Estado do Rio de Janeiro, 2018.

4. OLIVA, A. M.; ALVES FILHO, H.; SILVA, D. J.; GARCIA, C. R.. The spectral nodal method applied to multigroup SN neutron transport problems in one-dimensional geometry with fixedsource. Progress in Nuclear Energy, v. 22, p. 106-113, 2018.

5. BARROS, R. C. A Spectral Nodal Method for the Solution of Discrete Ordinates Problems in one and two Dimensional Cartesian Geometry. PhD Thesis, The University of Michigan, 1990.

6. BARROS, R. C.; LARSEN, E. W. A spectral nodal method for one-group X, Y-geometry discrete ordinates problems. Nuclear Science and Engineering, v. 111, p. 34-45, 1992.

7. DA SILVA, O. P. Um método de Matrix Resposta para cálculos de transporte multigrupos de energia na formulação de ordenadas discretas em meios não-multiplicativos. Tese de Doutorado, Universidade do Estado do Rio de Janeiro, 2018.

8. BENASSI, M. COTTA, R. M., Siewert, C. E. The PN Method for Radiative Transfer Problems with Reflective Boundary Conditions, Journal of Quantitative Spectroscopy and Radiative Transfer, vol. 30, pp. 547_553, 1983.

9. BARICHELlO, L. B; GARCIA, R. D. M.; SIEWERT, C. E. A Spherical Harmonics Solution For Radiative-Transfer Problems With Reflecting Boundaries And Internal Sources, Journal of Quantitative Spectroscopy and Radiative Transfer, v. 60, no. 2, p. 247-260, 1998. 
10. BARICHELLO, L. B.; SIEWERT, C. E.. A Discrete Ordinates Solution For A Non-Grey Model With Complete Frequency Redistribution. Journal of Quantitative Spectroscopy and Radiative Transfer, v. 62, p. 665-675, 1999.

11. VARGAS, R.;VILHENA, M. T. A closed-form solution for one-dimensional radiative condutive problem by the decomposition and LTSn methods. Journal of Quantitative Spectroscopy and Radiative Transfer, v. 61, p. 303-308, 1999. 